\title{
Antiradical and antidiabetic activity of Pleurotus ostreatus extracts
}

\author{
Mark Shamtsyan ${ }^{1}$ and Lea Pogačnik ${ }^{2, *}$ \\ ${ }^{1}$ St. Petersburg State Institute of Technology, Department of Technology of Microbiological \\ Synthesis, Moskovsky prospect, 26, St. Petersburg, Russia \\ ${ }^{2}$ University of Ljubljana, Biotechnical Faculty, Department of Food Science and Technology, \\ Jamnikarjeva 101, Ljubljana, Slovenia
}

\begin{abstract}
Mushrooms have been an important part of the diet for centuries. There are more than 2,000 different species of mushrooms found in the nature, but only 25 species are generally used in the human diet, and even fewer are grown commercially. One of the more popular mushrooms for cultivation and culinary use are mushrooms of the genus Pleurotus. The objectives of this study were: (i) preparation of isolates from the basidiomycetes Pleurotus ostreatus by sequential extraction with cold and hot water, extraction with hot water and extraction with hot alkali solution for determination of antiradical activity and (ii) preparation of water and $70 \%$ ethanol extracts for determination of antidiabetic activity. The antiradical activity of the extracts was determined using spectrophotometric method based on inhibition of free radical 2,2diphenyl-1-picrylhydrazyl. Antidiabetic activity was determined using $\alpha$ amylase inhibition assay with DNSA reagent and spectrophotometric determination. As a result of the work carried out, it was found that the preparations obtained by extraction exhibit antiradical activity. Regarding antidiabetic activity, all tested extracts showed considerable antidiabetic activity. Moreover, water extracts were inhibiting $\alpha$-amylase significantly more than ethanol extracts at higher concentrations of dry extract (50 and $100 \mathrm{mg} / \mathrm{mL}$ ), whereas at lower concentrations $(1 \mathrm{and} 10 \mathrm{mg} / \mathrm{mL}$ ) of ethanol extracts were more efficient than water ones.
\end{abstract}

\section{Introduction}

The universal mechanisms of development of many pathologies are associated with oxidative stress $[1,2]$. Being, on the one hand, protective adaptive response, oxidative stress can in many cases act as an inductor and a factor in the development of many oncological, cardiovascular, neurological diseases (Alzheimer's disease, Parkinson's disease), atherosclerosis, as well as participate in the aging process $[1,3]$. For these pathologies are characterized by an increased level of oxidized molecules (secondary radicals of lipids, proteins) in the systemic circulation and tissues. This is due to the excessive generation of active oxygen metabolites in conditions of oxidative stress and insufficient antioxidant defence of the body (enzymatic and / or non-enzymatic units) [1].

\footnotetext{
* Corresponding author: lea.pogacnik@bf.uni-lj.si
} 
To slow down pathological processes, it is extremely important to maintain the physiological level of oxidized molecules, the excess of which plays a negative role of attractants proinflammatory molecules and thus prolongs inflammatory reaction [2].

The balance of oxidized molecules is normally maintained by the components nonenzymatic link of the antioxidant system of the body. Many of these compounds a person receives with food.

Antioxidant properties are studied for the long time for synthetic substances and plant extracts [5]. Accents in publications of recent decades are focused on vegetable raw materials. As most researchers believe a promising combination of antioxidants, rather than isolated molecules, mushrooms attract close attention due to their high content of biologically active components. Connections with known antioxidant properties are found in bodies, mycelium, and mushroom cultures.

Antioxidant activity is associated with the presence of polysaccharides, tocopherols, phenolic compounds (phenolic acids and flavonoids), carotenoids, ergosterol, ascorbic acid, etc. $[1,2,4,5,7-9]$.

Finding of functional nutritional ingredients, which could not only help a person get rid of various kinds diseases, but also helped to increase his performance, make the body is more resistant to external adverse factors, and that most importantly, do not harm the body.

Thus, mushroom extracts are promising for use in as functional additives enriched with exogenous antioxidants. When included in the diet as an alternative food source, such extracts can prevent damage, caused by oxidative stress.

The composition of mushrooms includes various compounds that represent biological significance, from which a group of basic substances participating in the energy and plastic metabolism of the body. These compounds include proteins, carbohydrates, fats, and macronutrients. To the second class of nutrients includes micronutrients, including vitamins, minerals and other biologically irreplaceable for humans active substances present in small amounts.

Some classes of biopolymers found in fungi, such as proteins, polysaccharides, lipopolysaccharides and glycoproteins are classified as molecules that have a strong effect on the immune system [6]. Furthermore, recent studies suggest, that polysaccharides isolated from Pleurotus ostreatus may possess antidiabetic effects [10]. It was shown that extracts partially inhibit enzyme $\alpha$-amylase, so they could be used in the adjunctive treatment of type 2 diabetes [11].

Currently, preparations based on mushrooms, mainly from polysaccharides and, especially, $\beta$-glucans are widely used in medicine. Among the well-known ones are "Lentinan" (Lentinus edodes), "Pleoran" (Pleurotus ostreatus), "Befungin" (Inonotus obliquus), "Schizophyllan" (Schizophyllum commune), "Grifolan" (Grifola frondosa), "Krestin" (Trametes versicolor) and alsolarge variety of biologically active supplements [6]. For obtaining pharmacological preparations either the fruiting bodies of cultivated mushrooms, or preparations obtained on the basis of submerged cultivation of mushroom mycelia are used. However, the process of obtaining fruiting bodies is quite laborious and takes more time. Submerged cultivation allows to accelerate the growth of biomass by 7-10 times, as well as to regulate the composition complex of biologically active substances and carry out directed synthesis of target metabolites [12].

Mushrooms of the genus Pleurotus are ubiquitous in both temperate and and in tropical parts of the world [8] and have long attracted interest from manufacturers, due to their taste, relative simplicity cultivation and a rich set of biologically active substances. By the volume of production of fruit bodies Pleurotus ostreatus ranks third.

Aim of this study is to evaluate antioxidant, antiradical and antidiabetic activity of extracts obtained from the fruiting body biomass of Pleurotus ostreatus mushrooms 
purchased in Slovenia (Slovenian and Hungarian sample) and in St. Petersburg region of Russia.

\section{Materials and methods}

\subsection{Antiradical activity studies}

a) Preparation of P1: extraction with 5 volumes of $80 \%$ ethanol was carried out twice for $3 \mathrm{hrs}$. By the end of the extraction, the residue was filtered off on a vacuum filter. As a result, preparation $\mathrm{P} 1$ was biomass purified from most low-molecular impurities and lipids.

b) Preparation of P2: Obtained after treatment with ethanol preparation P1 was subjected to aqueous extraction, $600 \mathrm{~mL}$ of water was added for each $10 \mathrm{~g}$ of preparation Extraction at boiling temperature was carried out for 3 hours, repeated 3 times. After the extraction, the product was filtered on a vacuum filter and the extract was collected. The resulting extract concentrated by evaporation. High-molecular substances were precipitated with 5 volumes of $96 \%$ ethanol. The precipitate was filtered on a vacuum filter, then dried and crushed into powder, thus obtaining P2 preparation in the solid phase.

c) Preparation of P-3: The remaining after hot water extraction residue was studied as the preparation P-3.

d) Preparation of P2-1 and P2-2: to $10 \mathrm{~g}$ of obtained by ethanol treatment preparation P1, $200 \mathrm{~mL}$ of water was added, and water extraction was carried out for 24 hours with stirring at $30^{\circ} \mathrm{C}$. At the end of the extraction, the product was filtered on vacuum filter. Extract was collected, concentrated by evaporation and high-molecular substances were precipitated with 5 volumes of $96 \%$ ethanol. The resulted precipitate was filtered on a vacuum filter, then dried and crushed into powder, thus P2-1 was obtained Preparation P2-2 was isolated from the intermediate product obtained as a result of cold water extraction by sequential hot water extraction.

e) Preparation of P-4: To the residue of boiling aqueous extraction (preparation P-3) was added $1 \mathrm{M} \mathrm{NaOH}$ in a ration $600 \mathrm{~mL}$ to $10 \mathrm{~g}$. The extraction was carried out at boiling temperature for 3 hours. At the end of the extraction process, solution was neutralized with glacial acetic acid. The extract was filtered on a vacuum filter and was concentrated by evaporation followed by precipitation with a fivefold volume of $96 \%$ ethanol solution. The precipitate was filtered off on a vacuum filter, after which was dried and milled to obtain the powdered preparation P-4.

Assessment of antiradical activity of the studied preparations was carried out using a spectrophotometric method based on the inhibition of the free radical of 2,2-diphenyl-1picrylhydrazyl (DPPG). This method was first presented by M. Blois (1958) [13].

\subsection{Antidiabetic activity studies}

a) Fresh mushrooms of two different origin (Slovenia and Hungary, Figure 1) were freeze-dried (ALPHA 1-2 LD Plus, CHRIST, Germany) at $-50{ }^{\circ} \mathrm{C}$ and 0,12 mbar and milled into a powder.

b) $22,5 \mathrm{~mL}$ of extraction solvent $(\mathrm{dH} 2 \mathrm{O}$ or $70 \%$ ethanol) was added to $1,5 \mathrm{~g}$ of freezedried powder (solid-to-liquid ratio 1:15).

c) Extraction was carried in a water bath at $25^{\circ} \mathrm{C}$ for 30 minutes with constant shaking.

d) The insoluble matter was removed by a double 10 -minutes centrifugation at 4,000 rpm.

e) Furthermore, the solvent was removed by freeze-drying (water extracts) or rotavaporisation (ethanol extracts) to obtain the dried extracts. 
f) The concentrated extracts were prepared by addition of appropriate amount of DMSO (dimethyl sulfoxide) to obtain $100 \mathrm{mg}$ of dry extract per $\mathrm{mL}$ of concentrated extract.
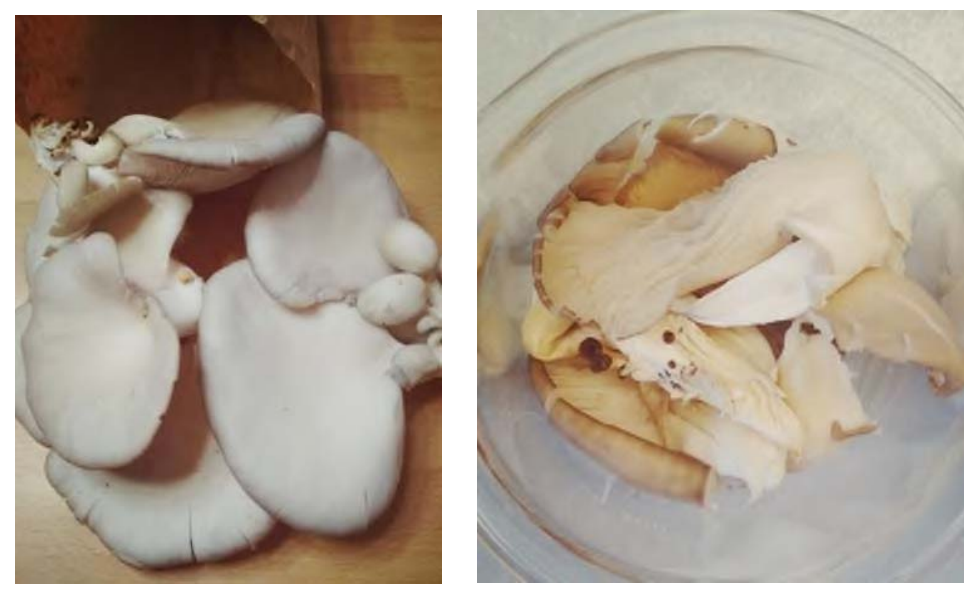

Fig. 1. Fresh samples of Pleurotus ostreatus from two different origin (left - Slovenia; right Hungary).

The anti- $\alpha$-amylase activity was assessed using $\alpha$-amylase inhibition assay, which measure the concentrations of reducing sugars spectrophotometrically at $540 \mathrm{~nm}$ using DNSA (3,5-dinitrosalicylic acid) as the colouring reagent [14]. The experimental procedure is described into details elsewhere [15].

\section{Results and discussion}

\subsection{Antiradical activity studies}

Results of the study of antiradical activity of the preparations obtained from basidiomycete Pleurotus ostreatus, are shown in Tables 1 and 2. In the Table 1 antiradical activity of the studied preparations is expressed as a percentage, reflecting the degree of discoloration of the DPPG solution.

Table 1. Inhibition of the free radical DPPH by preparations, obtained from Pleurotus ostreatus.

\begin{tabular}{|c|c|c|c|c|c|}
\hline \multirow{2}{*}{ No } & \multirow{2}{*}{$\begin{array}{c}\text { Variety of } \\
\text { preparation }\end{array}$} & \multicolumn{4}{|c|}{ Inhibition of DPPG radical, \% } \\
\cline { 3 - 6 } & & $0,625 \mathrm{mg} / \mathrm{ml}$ & $1,25 \mathrm{mg} / \mathrm{ml}$ & $2,5 \mathrm{mg} / \mathrm{ml}$ & $5,0 \mathrm{mg} / \mathrm{ml}$ \\
\hline 1 & P2-1 & $15,3 \pm 2,9$ & $18,2 \pm 1,3$ & $24,2 \pm 1,9$ & $30,0 \pm 3,2$ \\
\hline 2 & P2-2 & $42,1 \pm 3,0$ & $66,2 \pm 3,5$ & $80,1 \pm 1,7$ & $83,4 \pm 6,5$ \\
\hline 3 & P-3 & $32,9 \pm 2,8$ & $55,6 \pm 2,0$ & $75,9 \pm 1,5$ & $60,8 \pm 2,7$ \\
\hline 4 & P-4 & $32,8 \pm 3,2$ & $53,0 \pm 3,9$ & $75,9 \pm 4,6$ & $80,5 \pm 2,3$ \\
\hline
\end{tabular}

In the Table 2, the antiradical activity of the studied preparations is presented as "Effective concentration" - IC50, that is, this concentration of antioxidant, in which $50 \%$ of free radicals are neutralized. The lower the value of the IC50 parameter, the greater antiradical activity possesses a substance [5]. Generally, inhibition of the DPPG radical increases, with the increase of the concentration of preparations.

For the preparation P2-1 the value of the effective concentration was not calculated. But if we assume that the dependence of the antiradical activity on the concentration of 
preparation P2-1 has a linear form (the value of the approximation reliability R2 $=0.9668$ ), then the calculated value of IC50 for this preparation will be $10.8 \mathrm{mg} / \mathrm{ml}$. However, one cannot predict whether the linear relationship will persist with increasing concentration. As follows from the data presented in Tables 1 and 2, the P2-1 preparation exhibits the lowest antiradical activity compared to other preparations

Table 2. Effective concentration of the preparations obtained from Pleurotus ostreatus.

\begin{tabular}{|c|c|c|}
\hline No & Variety of preparation & IC50, $\mathrm{mg} / \mathrm{ml}$ \\
\hline 1 & P2-1 & - \\
\hline 2 & P2-2 & $0,72 \pm 0,04$ \\
\hline 3 & P-3 & $1,12 \pm 0,05$ \\
\hline 4 & P-4 & $1,14 \pm 0,07$ \\
\hline Control & Ascorbic acid & $0,0039 \pm 0,0002$ \\
\hline
\end{tabular}

Preparation P2-2 obtained by sequential extraction with cold and hot water, has the highest antiradical activity in comparison with other extracts. Thus, carrying out cold extraction before hot one can increase the value of the antiradical one.

The P-4 preparation exhibits antiradical activity comparable to the $\mathrm{P} 2$ preparation.

\subsection{Antidiabetic activity studies}

The results of antidiabetic activity of concentrated Pleurotus ostreatus extracts determined by $\alpha$-amylase activity are presented in Figure 2 (water extracts) and Figure 3 (70\% ethanol extracts).

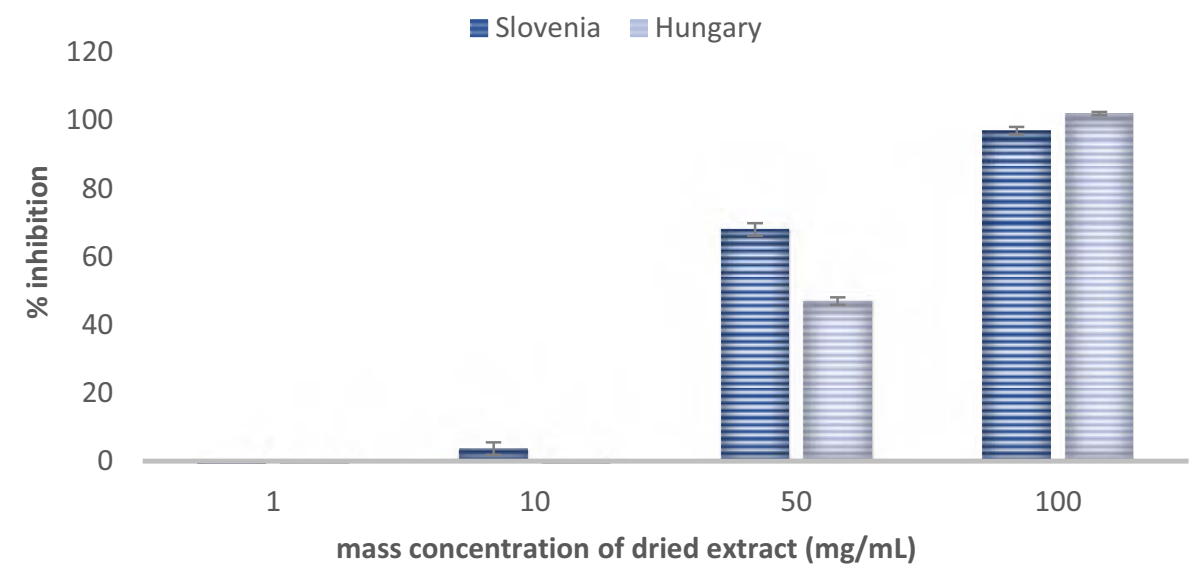

Fig. 2. Antidiabetic activity of water extracts of Pleurotus ostreatus from two different origin with different concentrations of dried extract.

The results obtained for water extracts show, that the lowest tested concentration (1 $\mathrm{mg} / \mathrm{mL}$ ) of dried extracts from Pleurotus ostreatus of both tested origins do not show any inhibition of $\alpha$-amylase, whereas 10 -times higher concentration $(10 \mathrm{mg} / \mathrm{mL})$ of Slovenian extract only slightly inhibits the enzyme (4\%). However, considerable inhibition was achieved with extracts' concentration $50 \mathrm{mg} / \mathrm{mL}$ for both extracts $(68 \%$ for Slovenian and $47 \%$ for Hungarian). Both extracts completely inhibited $\alpha$-amylase at the highest tested concentration $(100 \mathrm{mg} / \mathrm{mL})$. 


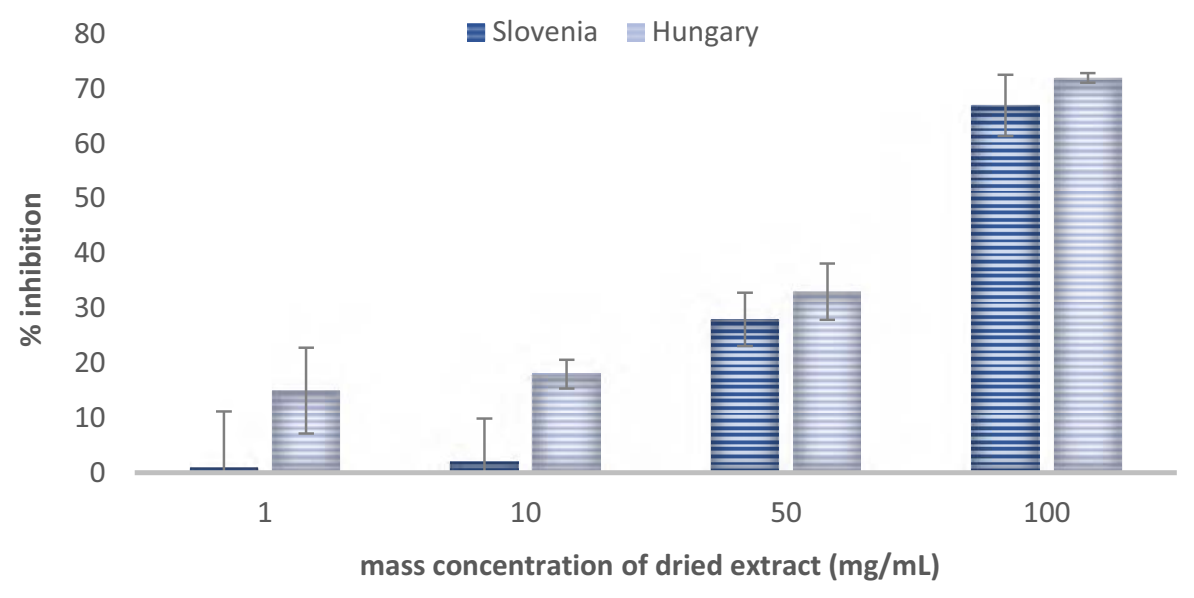

Fig. 3. Antidiabetic activity of $70 \%$ ethanol extracts of Pleurotus ostreatus from two different origin with different concentrations of dried extract.

The results obtained with $70 \%$ ethanol extracts show that all tested concentrations of dried extracts show inhibition of $\alpha$-amylase, Hungarian extracts being more effective for all tested concentrations. Compared to the water extracts the ethanol ones inhibit enzyme better at lower concentrations ( 1 and $10 \mathrm{mg} / \mathrm{mL}$ ), whereas the water extracts are more effective at higher tested concentrations (50 and $100 \mathrm{mg} / \mathrm{mL}$ ). At the highest tested concentration, the inhibition for both extracts was about $70 \%$ and at $50 \mathrm{mg} / \mathrm{mL}$ about $30 \%$ for both tested extracts, which is considerable less than results obtained with water extracts with the same concentration of dried extract.

\section{Conclusions}

It was shown by this study that Pleurotus ostreatus is a valuable source of bioactive substances, namely antioxidants and antidiabetic agents. In order to obtain highly active extracts, the extraction procedures have to be optimised, particularly regarding extraction solvents, temperature and duration of extraction. We showed that regarding antioxidants, it is important to perform a sequential extraction with cold and hot water.

For the anti- $\alpha$-amylase activities, both ethanol and water extracts showed dosedependent relationships between dry extract concentration and $\alpha$-amylase inhibition, which confirms the speculations that Pleurotus ostreatus and its extracts contain bioactive substances that can be used as anti- $\alpha$-amylase agents, which is in agreement with some previous studies $[10,11]$.

The research was carried out within the framework of the support of the bilateral Slovenian-Russian grant no BI-RU/19-20-046 and the Slovene Research Agency, Grant Number P4-0121.

\section{References}

1. C. Sanchez, Reactive oxygen species and antioxidant properties from mushrooms, Synthetic and Systems Biotechnology 2, 1, 13-22 (2017) 
2. I. Ferreira, Antioxidants in wild mushrooms, Current Medicinal Chemistry 16, 12, 1543-1560 (2009)

3. M.N. Alam, Review on in vivo and in vitro methods evaluation of antioxidant activity, Saudi Pharmaceutical Journal 21, 143-152 (2013)

4. I.P. Kaur, Screening methods for antioxidants-a review. Mini-Reviews in Medicinal Chemistry 6, 3, 305- 312 (2006)

5. P. Molyneux, The use of the stable free radical diphenylpicrylhydrazil (DPPH) for estimating antioxidant activity, Songklanakarin Journal of Science and Technology 26, 2, 211-219 (2004)

6. M.D. Asatiani et al., Higher Basidiomycetes mushrooms as a source of antioxidants (Progress in Mycology, Scientific Publishers [et al.], 2010)

7. T. Jayakumar, Antioxidant activity of the oyster mushroom, Pleurotus ostreatus, on CCl4-induced liver injury in rats, Food and Chemical Technolgy 44, 12, 989 (2006)

8. Polysaccharides from Basidiomycetes: a promising source for ummunostimulating and anticancerous activity (Biotechnology: Concepts and Applications, Narosa publishing house, 2008)

9. S.P. Wasser, Medicinal mushrooms as a source of antitumor and immunomodulating polysaccharides, Applies Microbiology and Biotechnology 60, 3, 258-274 (2002)

10. Y. Zhang, T. Hu, H. Zhou, Y. Zhang, G. Jin, Y. Yang, Antidiabetic effect of polysaccharides from Pleurotus ostreatus in streptozotocin-induced diabetic rats, International Journal of Biological Macromolecules 83, 126-132 (2016)

11. E. Tamboli, A. Bhatnagar, A. Mishra, Alpha-amylase inhibitors from mycelium of an oyster mushroom, Preparative Biochemistry \& Biotechnology 48/8, 693-699 (2018)

12. M. Shamtsyan, Potential to develop functional food products from mushroom bioactive compounds, Journal of Hygienic Engineering and Design 15, 51-59 (2016)

13. M. Blois, Antioxidant determinations by the use of a stable free radical, Nature 181, 1199-1200 (1958)

14. S. Keharom, R. Mahachai, S. Chanthai, The optimization study of alpha-amylase activity based on central composite design-response surface methodology by dinitrosalicylic acid method, Int. Food Res. J. 23, 10-17 (2016)

15. M.G.R. da Silva, M. Skrt, D. Komes, N. Poklar Ulrih, L. Pogačnik, Enhanced Yield of Bioactivities from Onion (Allium cepa L.) Skin and Their Antioxidant and Anti- $\alpha-$ Amylase Activities, Int. J. Mol. Sci. 21, 2909 (2020) 